\title{
ON REGULAR LOCAL RINGS ${ }^{1}$
}

\author{
K. R. MOUNT
}

This paper generalizes slightly a result of Kunz [1] and Nakai [2]. If $R>S$ are commutative rings with identity we introduce a module $D^{*}(R / S)$ defined as the quotient of the module $D(R / S)$ of $S$ differentials of $R$ by the submodule consisting of elements which are mapped to zero by every homomorphism of $D(R / S)$ having values in a finitely generated $R$ module. The characteristic exponent of a field is defined to be 1 if the field is of characteristic zero and to be $p$ if the characteristic of the field is $p$. The result is then: If $R$ is a local ring containing a field $k$ of characteristic exponent $p$ such that $D^{*}\left(R / k^{p}\right)$ is finitely generated, then the following conditions are equivalent: (i) $R$ is a regular local ring. (ii) $D^{*}\left(R / k^{p}\right)$ is free and if $x$ is an element of the completion of $R$ such that $x^{p}=0$ then $x=0$. (iii) $D^{*}\left(R / k^{p}\right)$ is free and if $x$ is an element of the form ring of $R$ such that $x^{p}=0$ then $x=0$. We remark that in characteristic zero regularity (under the finiteness condition) is equivalent to the freedom of $D^{*}(R / k)$ and in any case if the local ring is of the form $A_{q}$ where $A$ is a finitely generated integral domain and $q$ is a prime the second part of (ii) is automatically satisfied. (See Zariski and Samuel [4, p. 314].)

LEMMA 1. If $R>S$ are commutative rings with identity then there is one and only one module $D^{*}(R / S)$ (to within $R$-isomorphism) satisfying the conditions: (i) There is an $S$-derivation $d^{*}$ from $R$ to $D^{*}(R / S)$ such that the image of $d^{*}$ generates $D^{*}(R / S)$. (ii) If $h$ is an $S$ derivation from $R$ to a finitely generated $R$ module $M$ then there is an $R$ homomorphism $D^{*}(h)$ from $D^{*}(R / S)$ to $M$ such that $D^{*}(h) d^{*}=h$. (iii) If $f$ is an element of $D^{*}(R / S)$ then $h(f)=0$ for every homomorphism $h$ of $D^{*}$ to a finitely generated $R$ module if and only if $f=0$.

Proof. If $F(R / S)$ denotes the collection of elements of $D(R / S)$ annihilated by all $R$ homomorphisms to finitely generated $R$ modules, let $q$ denote the quotient map from $D(R / S)$ to $D(R / S) / F(R / S)$ $=D^{*}(R / S)$ and set $d^{*}=q d$ where $d$ is the derivation from $R$ to $D(R / S)$. If $h$ is an $S$ derivation from $R$ to a module $N$ denote by $D(h)$ the homomorphism from $D(R / S)$ satisfying $D(h) d=h$ and suppose $M$ is a second module with properties (i)-(iii) where $d \#$ denotes the derivation from $R$ to $M$ and $D \#(h)$ denotes the homomorphism assigned to a derivation from $R$ to $N$. If $b$ maps $M$ to a finitely

Received by the editors February 26, 1962.

1 This work was supported in part by NSF Grant G-9508. 
generated module then one checks easily that $D(b d \#)=b D(d \#)$ and hence if $f$ is an element of $F(R / S)$ we have $D(d \#)(f)=0$. Denote by $D^{*}(d \#)$ the homomorphism from $D^{*}(R / S)$ to $M$ satisfying the equation $D^{*}(d \#) q(f)=D(d \#)(f)$ for $f$ in $R$. If $x$ is an element of $D(R / S)$ with $D^{*}(d \#) q(x)=0$ then for a homomorphism $g$ from $D(R / S)$ to a finitely generated module $P$ it follows that $D \#(g d) D(d \#)=g$, thus $g(x)=0$ and $D^{*}(d \#)$ is an isomorphism.

Lemma 2. If $R$ is a local ring containing a field $K$ such that $D^{*}(R / K)$ is finitely generated, or if $M=D(R / K) / \cap m^{n} D(R / K)$ is finitely generated then $M=D^{*}(R / K)$ ( $m$ the maximal ideal of $R$ ).

Proof. First $D^{*}(R / K)$ is clearly hausdorff. Denote by $h$ the quotient map from $R$ to $R / m=L$ and note that $D^{*}(L / K)=D(L / K)$, using Lemma 1 . Let $A$ be the submodule of $R / m \otimes D^{*}(R / K)$ generated by the elements of the form $1 \otimes d^{*} x$ for $x$ in $m$ and set $D \#(L / K)$ $=\left[R / m \otimes D^{*}(R / K)\right] / A$. Define a derivation $d \#$ from $R / m$ to $D \#(L / K)$ by $d \#(x)=\mathrm{Cl}\left(1 \otimes d^{*} x\right)$ where $\mathrm{Cl}(y)$ denotes the coset determined by the element $y$. If $f$ is a derivation (over $K$ ) from $R / m$ to $M$, a finitely generated $L$ module, then $f h$ is a derivation of $R$ to $M$ and the map $1 \otimes D^{*}(f h)$ induces a homomorphism $D \#(f)$ from $D \#(L / K)$ to $M$ such that $D \#(f) d \#(x)=f(x)$. Since $D \#(L / K)$ is finitely generated it satisfies (i)-(iii) of Lemma 1 and thus $D \#(L / K)=D^{*}(L / K)=D(L / K)$. Denote by $R(n)$ the ring $R / m^{n}$ and by $m(n)$ the maximal ideal of $R(n)$ and note that we have the exact sequence $m(n) / m(n)^{2}$ $\rightarrow R(n) / m(n) \otimes D(R(n) / K) \rightarrow D(L / K) \rightarrow 0$ (Nakai [2, Proposition 9]). It follows easily that $D(R(n) / K)$ is finitely generated. Now consider an element $z$ of $F(R / K)$ and note that if $h(n)$ denotes the quotient map from $R$ to $R(n)$ and if $d(n)$ is the $K$ derivation from $R(n)$ to $D(R(n) / K)$ then $D(d(n) h(n))$ is a homomorphism from $D(R / K)$ to $D(R(n) / K)$ with kernel contained in $m^{n-1} D(R / K)$ (Nakai [2, Proposition 9]) and thus $z$ is an element of $\cap m^{n} D(R / K)$. If $M$ is finitely generated then one checks easily that it satisfies conditions (i)-(iii) of Lemma 1.

If $R$ is a local ring with maximal ideal $m$ and $M$ is a hausdorff $R$ module in the $m$-adic topology denote by $\operatorname{Co}(M)$ the completion of $M$.

Lemma 3. If $R$ is a local ring containing a field $K$ with $D^{*}(R / K)$ finitely generated then the completion of $D^{*}(R / K)$ is isomorphic to $D^{*}(\mathrm{Co}(R) / K)$.

Proof. It will suffice to show that there is an isomorphism from the module $D^{*}(R / K) / m^{n} D^{*}(R / K)$ to $D^{*}(\operatorname{Co}(R) / K) / m^{n} D^{*}(\operatorname{Co}(R) / K)$ which commutes with the quotient maps. Denote by $D(n)^{*}$ the 
module $D^{*}(R / K) / m^{n} D^{*}(R / K)$ and by $C(n)^{*}$ the module $D^{*}(\operatorname{Co}(R) / K) / m^{n} D^{*}(\operatorname{Co}(R) / K)$. Let $p(n+1 / n)$ and $q(n+1 / n)$ represent the maps from $D(n+1)^{*}$ to $D(n)^{*}$ and from $C(n+1)^{*}$ to $C(n)^{*}$ respectively and denote by $p(n)$ and $q(n)$ the quotient maps from $D^{*}(R / K)$ to $D(n)^{*}$ and from $D^{*}(\operatorname{Co}(R) / K)$ to $C(n)^{*}$. If $c^{*}$ denotes the derivation from $\operatorname{Co}(R)$ to $D^{*}(\operatorname{Co}(R) / K)$ then the derivation $d(n) \#=q(n) c^{*}$ gives rise to a homomorphism $D(d(n) \#)$ from $D(R / K)$ to $C(n)^{*}$ such that $D(d(n) \#) d=d(n) \#$. If $f$ is an element of $m^{n} D(R / K)$ then $D(d(n) \#)(f)=0$ and thus there is a homomorphism $J(n)$ from $D(n) *$ to $C(n) *$ satisfying the equation $(J(n) p(n))(r(g))=D(d(n) \#)(g)$ for $g$ in $D(R / K)$ and $r$ the quotient map from $D(R / K)$ to $D^{*}(R / K)$. Suppose $h$ is in $\operatorname{Co}(R)$, write $h$ in the form $h=h^{\prime}+h^{\prime \prime}$ with $h^{\prime}$ in $R$ and $h^{\prime \prime}$ in $m^{n+1} \operatorname{Co}(R)$ and set $e(n)[h]=q(n) d^{*}\left(h^{\prime}\right)$. The map $e(n)$ defines a $K$ derivation from $\operatorname{Co}(R)$ to $D(n)^{*}$ where we consider $D(n)^{*}$ as a $\mathrm{Co}(R)$ module by setting $h \cdot u=h^{\prime}$ for $h^{\prime}$ as above. Denote by $C(v)$ the homomorphism from $D(\operatorname{Co}(R) / K)$ to $P$ determined if $v$ is a $K$ derivation from $\operatorname{Co}(R)$ to $P$. If $u$ is an element of $m^{n} D(\operatorname{Co}(R) / K)$ then $C(e(n))(u)$ is an element of $m^{n} D(R / K)$ whence there is a homomorphism $H(n)$ from $C(n)$ * to $D(n) *$ with $H(n)(q(n) s(f))=C(e(n))(f)$ for $s$ the quotient map from $D(\operatorname{Co}(R) / K)$ to $D^{*}(\operatorname{Co}(R) / K)$. For $x$ in $R$ we have $(H(n) J(n))\left(p(n) d^{*} x\right)=p(n) d^{*} x$ and hence $J(n)$ is a monomorphism. If $x$ is in $\operatorname{Co}(R)$ then writing $x=x^{\prime}+x^{\prime \prime}$ with $x^{\prime \prime}$ in $m^{n+1} \operatorname{Co}(R)$ and $x^{\prime}$ in $R$ we have that $c^{*}(x)=c^{*}\left(x^{\prime}\right)$ modulo $m^{n} D^{*}(\operatorname{Co}(R) / K)$ from which it follows that $J(n)$ is onto. To complete the assertion we need only show that $J(n) p(n+1 / n)=q(n+1 / n) J(n+1)$ and it suffices to show this for the elements of the form $p(n+1) r d x$ which one checks easily.

As a consequence we have that if $R=K\left[\left[X_{1}, \cdots, X_{n}\right]\right]$ with $\left[K ; K^{p}\right]<\infty$ then $D^{*}\left(R / K^{p}\right)$ is free on the basis $d^{*} X_{i}$ and $d^{*} Y_{j}$ where $Y_{j}$ is a $p$ basis of $K$ over $K^{p}$. This follows by completing $K\left[X_{1}, \cdots, X_{n}\right]_{X}$ where $X$ is the ideal generated by the $X_{i}$. Also note that if $R$ is any local ring containing a field $K$ such that $D^{*}(R / K)$ is finitely generated and if $M$ is a hausdorff $R$ module then any homomorphism from $D(R / K)$ to $M$ annihilates $F(R / K)$.

Proposition 1. Let $R$ be a local ring containing a field $K$ such that $D^{*}(R / K)$ is finitely generated. If $R^{\prime}>R$ with $R^{\prime}$ regularly quasi-finite over $R$ then $D^{*}\left(R^{\prime} / K\right)$ is finitely generated.

(For definitions see $[3]$ ).

Proof. Suppose $R^{\prime}=R\left[x_{1}, \cdots, x_{n}\right]$ and assume $R^{\prime}=R_{m}^{\prime \prime \prime}$ where $m^{\prime \prime}$ is a maximal ideal of $R^{\prime \prime}$. Denote by $N^{\prime \prime}$ the image of $R^{\prime \prime}$ under the map $d \cdot k$ where $k$ is the inclusion of $R^{\prime \prime}$ into $R^{\prime}$ and let $g$ denote 
the induced map from $D\left(R^{\prime \prime} / K\right)$ to $D^{*}\left(R^{\prime} / K\right)$. The image of $g$ is spanned by the restriction of $g$ to the set $(d \cdot k)(R)$ and by the $g d x_{i}$. We note first that $D^{*}\left(R^{\prime} / K\right)$ is a hausdorff $R^{\prime}$ module and thus is a hausdorff $R$ module. The map $g(d \cdot k)$ restricted to $R$ is thus a $K$ derivation of $R$ to a hausdorff $R$ module and hence the image of $g$ is generated by a homomorphic image of $D^{*}(R / K)$ and by the $g d x_{i}$ whence $N^{\prime \prime}$ is finitely generated. Now suppose $f$ is in $R^{\prime}$. There is an element $n$ of $R^{\prime \prime}, n$ not in $m^{\prime \prime}$, such that $n f$ lies in $R^{\prime \prime}$, thus $d^{*}(n f)$ $=d^{*}(n) \cdot f+n d^{*}(f)$, hence $d^{*}(f)=(1 / n) \cdot h$ where $h$ is in the image of $g$ so $D^{*}\left(R^{\prime} / K\right)$ is finitely generated.

Lemma 4. Suppose $f$ is an epimorphism of the local ring $R$ to the local ring $R^{\prime}$ such that $R$ contains a field $K$ with $D^{*}(R / K)$ and $D^{*}\left(R^{\prime} / K\right)$ finitely generated. If $A=$ kernel $(f)$ then we have the exact sequence $(R / A) \otimes A \rightarrow R / A \otimes D^{*}(R / K) \rightarrow D^{*}\left(R^{\prime} / K\right) \rightarrow 0$.

Proof. Denote by $B$ the submodule of $R / A \otimes D^{*}(R / K)$ generated by the elements of the form $1 \otimes d^{*} a$ where $a$ is in $A$ and set $M=\left[R / A \otimes D^{*}(R / K)\right] / B$. If $h$ is the quotient map of $R / A \otimes D^{*}(R / K)$ onto $M$ we set $g(x)=h\left(1 \otimes d^{*} x^{\prime}\right)$ where $f\left(x^{\prime}\right)=x$ and note that this defines a map of $R / A$ into $M$ which is independent of the representation $x^{\prime}$ and is a $K$ derivation of $R / A$. The induced homomorphism $H^{*}=D^{*}(g)$ from $D^{*}\left(R^{\prime} / K\right)$ to $M$ is such that $H^{*}\left(d^{*} x\right)=g(x)$ for $x$ in $R / A$. On the other hand the module $D^{*}\left(R^{\prime} / K\right)$ is finitely generated as an $R$ module and the map $d^{*} f$ from $R$ to $D^{*}\left(R^{\prime} / K\right)$ is a $K$ derivation of $R$, thus $D^{*}\left(d^{*} f\right)$ maps $D^{*}(R / K)$ to $D^{*}\left(R^{\prime} / K\right)$ such that $D^{*}\left(d^{*} f\right)\left(d^{*} x\right)=d^{* *} f(x)$ for $x$ in $R$. It follows that $1 \otimes D^{*}$ carries $M$ into $D^{*}\left(R^{\prime} / K\right)$ by $\left(1 \otimes D^{*}\right)(x \otimes y)=x D^{*}(y)$ with $\left(1 \otimes D^{*}\right)(d z)=0$ for $z$ in $A$. There is thus a map $E^{*}$ from $M$ to $D^{*}\left(R^{\prime} / K\right)$ and one need only check that $H^{*} E^{*}$ and $E^{*} H^{*}$ are the identity.

Lemma 5. Let $R=K\left[\left[X_{1}, \cdots, X_{n}\right]\right]$ with $\left[K ; K^{p}\right]<\infty$ where $p$ is the characteristic exponent of $K$, suppose $A$ is an ideal of $R$ and assume that $D(A)<A$ for every $K^{p}$ derivation of $R$ into $R$. If $A \neq 0$ then (i) there is an element $x$ of $R$ such that $x$ is not in $A$ but $x^{p}$ is in $A$, or $A=R$ and (ii) there is an element $x$ of the form ring of $R / A$ with $x \neq 0$ and $x^{p}=0$ or $A=R$.

Proof. Choose a $p$-basis for $K$ over $K^{p}$ say $y_{1}, \cdots, y_{r}$. If $Q$ is a power series in $R$ we define the total degree of $Q$ to be the pair $(u, v)$ where $u$ is the subdegree of $Q$ and $v$ is the degree of the leading form of $Q$ considered as a polynomial in the $y_{j}$. Order the total degrees lexicographically and choose an element $P$ of $A$ of least total degree $(a, b)$ and assume $b \neq 0$. Since $b$ is nonzero the partial derivative of $P$ 
with respect to one of the $y_{j}$ occurring in the leading form $L(P)$ of $P$ lies in $A$ and reduces the total degree, thus no $y_{j}$ may occur in $L(P)$ and $L(P)$ is in $K^{p}\left[X_{1}, \cdots, X_{n}\right]$. Now consider any one of the indeterminates $X_{i}$ and note that the subdegree of $P$ will be reduced by differentiating with respect to $X_{i}$ unless the exponent to which $X_{i}$ occurs in a given monomial of $L(P)$ is of the form $s p$. We therefore have assertion (ii). If $Q$ is in $R$ we may write it in the form $Q=\sum Q_{a}$ where $a$ ranges over the collection of all the subsets of $T=\langle 1, \cdots, n\rangle$ (including the empty set) and $Q_{a}$ is the sum of those monomials $M$ of $Q$ such that $X_{i}$ appears in $M$ with exponent of the form $s p$ for those and only those $i$ in $a$. We now denote by $B_{j}$ the operation $X_{j} \partial / \partial X_{j}$ and note that $B_{j}$ maps $A$ into itself and that $B_{j}$ is zero on the monomials of $A$ in which $X_{j}$ occurs with an exponent which is a multiple of $p$. The application of $B_{j}(p-1)$ times is the identity on any monomial which does not have $X_{j}$ occurring with exponent a multiple of $p$. If $Q$ is in $A$ then applying $B_{n}(p-1)$ times and subtracting the result from $Q$ we have that $\sum Q_{a}$ is in $A$ where the sum runs over those subsets of $T$ which contain $n$ and by induction we have that $Q_{T}$ is in $A$. Using (i) we have that there are elements of $A$ such that $Q_{T} \neq 0$. Let $W$ denote the collection of elements of $A$ of least subdegree which lie in $K\left[\left[X_{1}^{p}, \cdots, X_{n}^{p}\right]\right]$ and let $t$ be the least degree of the elements of $W$ considered as polynomials in the $y_{j}$ with coefficients in $K^{p}\left[\left[X_{1}^{p}, \cdots, X_{n}^{p}\right]\right]$. To prove assertion (i) it suffices to show that $t=0$. Note, however, that the set $W$ remains fixed under the partial derivatives with respect to the $y_{j}$ from which the assertion is immediate.

THEOREM. If $R$ is a local ring containing a field $k$ of characteristic exponent $p$ such that $D^{*}\left(R / k^{p}\right)$ is finitely generated then the following conditions are equivalent: (i) $R$ is regular, (ii) $D^{*}\left(R / k^{p}\right)$ is free and if $x$ is an element of $\operatorname{Co}(R)$ such that $x^{p}=0$ then $x=0$, (iii) $D^{*}\left(R / k^{p}\right)$ is free and if $x$ is an element of the form ring of $R$ such that $x^{p}=0$ then $x=0$.

Proof. We first remark that if $k<K$ then in characteristic $p$ (nonzero) we have that $D^{*}\left(R / k^{p}\right)=D^{*}\left(R / K^{p}\right)$ and in any case by Lemma 3 we may suppose that the ring $R$ is complete. We may therefore assume in nonzero characteristic that $k=K$ is a field of coefficients of $R$. Consider the map $1 \otimes d \#$ carrying the module $\mathrm{m} / \mathrm{m}^{2}$ into $R / m \otimes D^{*}(R / K)$. To prove $1 \otimes d \#$ is an injection it suffices to prove the assertion for $R / m^{2}=R^{*}=K+m^{*}$ where $m^{*}$ is the maximal ideal of $R^{*}$. The projection $g$ of $R^{*}$ onto $m^{*}$ is a $K$ derivation to a finitely generated $R^{*}$ module thus $D^{*}(g)$ maps $D^{*}(R / K)$ to $m^{*}$ such that $D^{*}(g) d \# m=g(m)=m$ for $m$ in $m^{*}$, thus $1 \otimes d \#$ is an injection. The map 
$1 \otimes d^{*}$ from $m / m^{2}$ to $R / m \otimes D^{*}\left(R / K^{p}\right)$ is such that if $x$ is in $m$ and $1 \otimes d^{*}(x)=0$ then $1 \otimes D^{*}(d \#)\left(1 \otimes d^{*}\right)(x)=0$ which implies $x$ is in $m^{2}$ by the above. Thus we have an exact sequence $0 \rightarrow m / m^{2}$ $\rightarrow R / m \otimes D^{*}\left(R / K^{p}\right) \rightarrow D^{*}\left(K / K^{p}\right) \rightarrow 0$ in nonzero characteristic. Similarly in zero characteristic we may replace $K^{p}$ by $k$ in the above sequence. In nonzero characteristic we have that $\left[K: K^{p}\right]<\infty$ and a basis for $D\left(K / K^{p}\right)$ is given by the $d Y_{j}$ where the $Y_{j}$ are a $p$-basis for $K$ over $K^{p}$, and thus if $m_{i}, 1 \leqq i \leqq n$ is a minimal system of generators for the maximal ideal of $R$ the elements $d^{*} m_{j}$ and $d^{*} Y_{i}$ are a basis for the module $D^{*}\left(R / K^{p}\right)$. In characteristic zero the $d^{*} m_{j}$ are a subset of a basis for $D^{*}(R / k)$. Let $f$ be a map from $K\left[\left[X_{1}, \cdots, X_{n}\right]\right]$ onto $R$ carrying $K$ onto $K$ and $X_{i}$ onto $m_{i}$ where the $X_{i}$ are indeterminates. Set $N=$ kernel $(f)$ and assume $x$ is in $N$. We have the equation $0=d^{*}(f(x))=\sum f\left(\partial x / \partial X_{i}\right) d^{*} m_{i}+\sum f\left(\partial x / \partial Y_{j}\right) d^{*} Y_{j}$ in characteristic $p \neq 0$ and since $D^{*}\left(R / K^{p}\right)$ is free all the partials of $x$ must be in $N$ which implies $N=0$ under the assumptions (ii) and (iii) by Lemma 5 . In the case of characteristic zero we have that the partials with respect to the $X_{i}$ all are in $N$ since the $d^{*} m_{i}$ can be extended to a basis and we may again apply Lemma 5 .

\section{BIBLIOGRAPHY}

1. E. Kunz, Differentialformen inseparable algebraischer Funktionenkörper, Math. Z. 76 (1961), 56-74.

2. Y. Nakai, On the theory of differentials in commutative rings, J. Math. Soc. Japan 13 (1961), 63-84. 1953.

3. P. Samuel, Algebre locale, Memor. Sci. Math., No. 123, Gauthier-Villars, Paris,

4. O. Zariski and P. Samuel, Commutatative algebra, Vol. II, Van Nostrand, Princeton, N. J., 1960.

NORTHWESTERN UNIVERSITY 